\title{
CONDIÇÃO PERIODONTAL E HÁBITOS DE HIGIENE BUCAL DE IDOSOS NÃO INSTITUCIONALIZADOS, ATENDIDOS EM GRUPOS DE CONVIVÊNCIA DA CIDADE DE SALVADOR
}

\author{
Anderson Luiz de Almeida Chagas Cirurgião-dentista \\ Gabriel De Sousa Petró Mestre e doutora em Periodontia. \\ Professora Adjunta da Escola \\ Bahiana de Medicina e Saúde Pública \\ Mônica Dourado \\ Mestre e doutora em Periodontia. \\ Professora Adjunta da Escola \\ Bahiana de Medicina e Saúde Pública
}

\begin{abstract}
Resumo
Objetivo: O objetivo deste estudo foi avaliar a condição periodontal e os hábitos de higiene bucal dos idosos participantes de grupos de convivência não institucionalizados na cidade de Salvador. Metodologia: Foram incluídos na pesquisa 113 indivíduos de ambos os gêneros, com pelo menos 60 anos de idade, dos grupos de convivência de idosos localizados nos nove centros sociais urbanos mantidos pelo governo do estado da Bahia na cidade de Salvador. Os indivíduos responderam a um questionário dirigido onde foram coletadas informações sobre: idade, raça, nível de escolaridade, renda, pressão arterial, percepção da higiene bucal. Também foram submetidos a exame periodontal envolvendo número de dentes presentes, profundidade de sondagem, nível de inserção clínica, recessão gengival e índice de sangramento à sondagem. Resultados: $\mathrm{O}$ gênero feminino foi prevalente em todos os centros visitados, a idade média foi de 70,6 anos, e a renda média de até 1 salário mínimo. A prevalência de desdentados foi de $25,7 \%$, enquanto que $80,5 \%$ dos dentados apresentavam perda de inserção clínica. Conclusão: É clara a necessidade da elaboração de politicas públicas direcionadas aos idosos de baixa renda.
\end{abstract}

Palavras-chave: Saúde do idoso. Higiene bucal. Saúde bucal.

\section{PERIODONTAL CONDITION AND ORAL HYGIENE AND HABITS OF NON- INSTITUTIONALIZED ELDERLY PEOPLE, ASSISTED BY GROUPS OF COEXISTENCE IN THE CITY OF SALVADOR}

\begin{abstract}
Purpose: The purpose of this study was to evaluate the periodontal condition and oral hygiene habits of the elderly participants in groups of non-institutionalized living in the city of Salvador. Methodology: We included 113 individuals in search of both genders, at least 60 years of age, groups of living of the elderly located in nine municipal social centers maintained by the government of Bahia state in the city of Salvador. The individuals answered a questionnaire about where they were collected: age, race, education level, income, blood pressure, awareness of oral hygiene. They also underwent periodontal examination involving the number of teeth present, depth of sample, level of clinical integration, gingival recession and bleeding on probing index. Results: The female was prevalent in all the centers visited, the average age was 70,6 years and average income of up to 1 minimum wage. The prevalence of edentulous was $25,7 \%$ while $80,5 \%$ of the teeth had loss of clinical integration. Conclusion: It is a clear need for the development of public policies targeted to low-income elderly.
\end{abstract}

Keywords: Health of the elderly; Oral hygiene; Oral health. 
Chagas et al. Condição periodontal e hábitos de higiene bucal de idosos não institucionalizados, atendidos em grupos de convivência da cidade de Salvador.

\section{INTRODUÇÃO}

Atualmente estima-se que existam mais de 500 milhões de idosos no mundo, sendo que no Brasil são aproximadamente 15 milhões. A projeção da Organização Mundial de Saúde (OMS) para a população de idosos é de um crescimento de cerca de 16 vezes até 2025 , contra apenas 6 vezes da população em geral. ${ }^{(1)}$ A diminuição nas taxas de mortalidade, os avanços da medicina e consequente longevidade populacional, seguidas de um menor índice de natalidade, contribuem para uma transformação drástica na estrutura etária brasileira, levando de forma direta para a um envelhecimento coletivo do país.

A constituição e a política nacional do idoso (Lei 8.842/94) garantem a cada um deles o direito de não ficar em asilo se precisar de assistência médica hospitalar permanente, assistência integral a saúde pela rede pública, acesso a remédios, prótese e órteses e preferência no atendimento em órgãos públicos e particulares entre outros direitos. ${ }^{(2)}$

A preocupação com o indivíduo dentro de uma visão global e não só com os dentes é base da formação dos profissionais de hoje. A falta de percepção da necessidade de tratamento odontológico é muito comum, não somente entre os idosos, suas famílias e cuidadores, mas também entre muitos profissionais de saúde. ${ }^{(1)}$ É importante no atendimento ao paciente idoso, realizar uma avaliação mais pormenorizada, checando indícios de alterações importantes na sua fisiologia, orientando-os a procurar um profissional especialista, objetivando o equilíbrio das condições de saúde.

Apesar da predisposição política demonstrada pelo Governo Federal em atender às necessidades básicas dos idosos, tais iniciativas ainda são modestas frente à carência encontrada em diversas áreas, principalmente na saúde pública. De acordo com dados do IBGE ${ }^{(3)}$ existem aproximadamente 165.036 habitantes na faixa etária acima dos 60 anos na cidade de Salvador. Atualmente, há alguns projetos e ações diretamente ligados à saúde do idoso, como campanhas de prevenção a doenças sistêmicas de maior recorrência como diabetes mellitus e hipertensão arterial. Porém, não existem ações específicas de atenção a saúde bucal dos idosos, o que resultaria em um melhor controle destas alterações, haja vista existirem comprovações científicas da relação direta entre doença periodontal e exacerbação destas alterações sistêmicas.

Sabendo-se da importância sócio-econômica desta parcela da população, principalmente da de baixa renda, que na sua maioria são mantenedores dos seus lares e muitos ainda se mantém no mercado informal de trabalho, se faz necessário políticas públicas 
Chagas et al. Condição periodontal e hábitos de higiene bucal de idosos não institucionalizados, atendidos em grupos de convivência da cidade de Salvador.

que aumentem a condição de saúde desta população. Para isso, seria preciso ampliar ações preventivas, que se mostram pouco onerosas e de grande ganho em qualidade de vida e consequente diminuição de gastos públicos em saúde.

Em idosos, a perda da função mastigatória está diretamente relacionada com a perda dos dentes. Com o passar dos anos ocorrem várias modificações no organismo, principalmente no sistema digestivo, começando pela cavidade bucal, onde observa-se uma retração da gengiva, diminuição das papilas gustativas, da saliva e da ação de enzimas que participam do processo digestivo. $\mathrm{O}$ estilo de vida moderno, o ritmo de vida cada vez mais acelerado das grandes cidades, somado à influência da publicidade, são fatores que contribuem para mudar a alimentação. Desta forma, substitui-se alimentos ricos em fibras e nutrientes pelos industrializados, ricos em gordura e carboidratos refinados, que fermentam e facilitam a proliferação bacteriana na cavidade bucal, limitando a obtenção de uma dieta rica e variada em nutrientes indispensáveis para manutenção do sistema imunológico. ${ }^{(4)}$

Um componente importante na avaliação de saúde dos indivíduos é o reconhecimento de alterações fisiológicas, que deveriam ser observadas pelo cirurgião dentista mediante uma minuciosa anamnese e exame clínico pormenorizado, seguido de uma ficha clínica direcionada para o idoso, sendo necessário também existir um parâmetro mínimo do seu estado cognitivo e emocional para desenvolvimento de um plano de tratamento específico. ${ }^{(5)}$

Com o desenvolvimento de pesquisas na periodontia que passaram a entender a periodontite como uma doença de repercussão sistêmica e também influenciada por condições e alterações gerais, percebeu-se a sua relação com condições patológicas cardiovasculares, diabetes, doenças respiratórias, entre outros. ${ }^{(6)}$ Portanto, sendo essas doenças, assim com a periodontite, mais prevalentes em populações adultas a idosas, conhecer o estado periodontal, além dos próprios cuidados de higiene bucal desta população, se torna importante no intuito de se promover políticas de saúde adequadas, as quais tomam uma importância e ultrapassam o âmbito odontológico.

Dentro deste contexto, o presente estudo teve como objetivo avaliar a condição periodontal e os hábitos de higiene bucal dos idosos participantes de grupos de convivência não institucionalizados na cidade de Salvador, para orientar uma estratégia de atendimento em saúde bucal para população idosa de baixa renda. 
Chagas et al. Condição periodontal e hábitos de higiene bucal de idosos não institucionalizados, atendidos em grupos de convivência da cidade de Salvador.

\section{CASUÍSTICA E MÉTODOS}

Foram incluídos na pesquisa indivíduos de ambos os gêneros, com pelo menos 60 anos de idade, dos grupos de convivência de idosos localizados nos nove centros sociais urbanos mantidos pelo governo do estado da Bahia na cidade de Salvador. A população total envolvida nestes centros é de 376, sendo que destes 113 fizeram parte direta do trabalho, representados por 5 do gênero masculino e 108 do gênero feminino que após apresentação dos pesquisadores, decidiram participar de forma espontânea. Em seguida os participantes assinaram o termo de consentimento livre e esclarecido, conforme aprovado pelo protocolo ${ }^{\circ}$ 141/2008 do Comitê de Ética em Pesquisa da Escola Bahiana de Medicina e Saúde Pública (EBMSP).

$\mathrm{Na}$ chegada a cada unidade, e sem agendamento prévio, foi apresentado documento de acesso expedido pela Secretaria de Desenvolvimento Social e Combate a Pobreza (SEDES), evitando qualquer tipo de expectativa por parte dos participantes com a chegada dos pesquisadores. Uma breve explanação sobre que se tratava a pesquisa era realizada, e os voluntários se dirigiam aos pesquisadores para aferição da pressão arterial (PA), respondiam ao questionário dirigido, e passavam por exame periodontal realizado por um único examinador calibrado (kappa > 0.8). A aferição da PA foi realizada com equipamento manual (BIC ML 040-2003) e os resultados obtidos foram classificados em níveis de hipertensão arterial sistêmica (HAS). ${ }^{(7)}$

Os exames periodontais envolveram a medição da profundidade de sondagem (PS), aumento ou recessão gengival e Nível de Inserção Clínica (NIC) em seis sítios de cada dente. ${ }^{(8)}$

Foram utilizados kits contendo sonda milimetrada tipo williams e nabers (TRINITY Av. Dr. Felipe Pinel, no 3455, Jaraguá, São Paulo-SP, Brasil) e espelho clínico n ${ }^{\circ} 5$. Ao final dos exames de todos os participantes de cada centro, foi realizada uma palestra informativa evidenciando métodos eficientes e corretos para a realização da higiene bucal. Algumas unidades a critério da coordenação, solicitaram que a palestra dos pesquisadores fosse realizada em outro momento.

\section{RESULTADOS}

O grupo estudado era composto por 113 idosos, com idade entre 60 e 85 anos, media de 70,6 anos, sendo $5(4,5 \%)$ do gênero masculino e 108 (95,5\%) do gênero feminino, 
Chagas et al. Condição periodontal e hábitos de higiene bucal de idosos não institucionalizados, atendidos em grupos de convivência da cidade de Salvador.

distribuídos segundo classificação dos pesquisadores em 67 (59,3\%) melanodermas, 27 $(23,9 \%)$ leucodermas e $19(16,8 \%)$ faiodermas, destes $82(72,6 \%)$ nascidos no interior e 31 $(27,4 \%)$ na capital, atualmente todos residindo na capital.

A totalidade do grupo pratica atividades físicas pelo menos 2 vezes por semana e só foram encontrados $6(5,3 \%)$ fumantes declarados.

Com relação a PA, a maioria do grupo foi classificada como portador de hipertensão controlada (Figura 1).

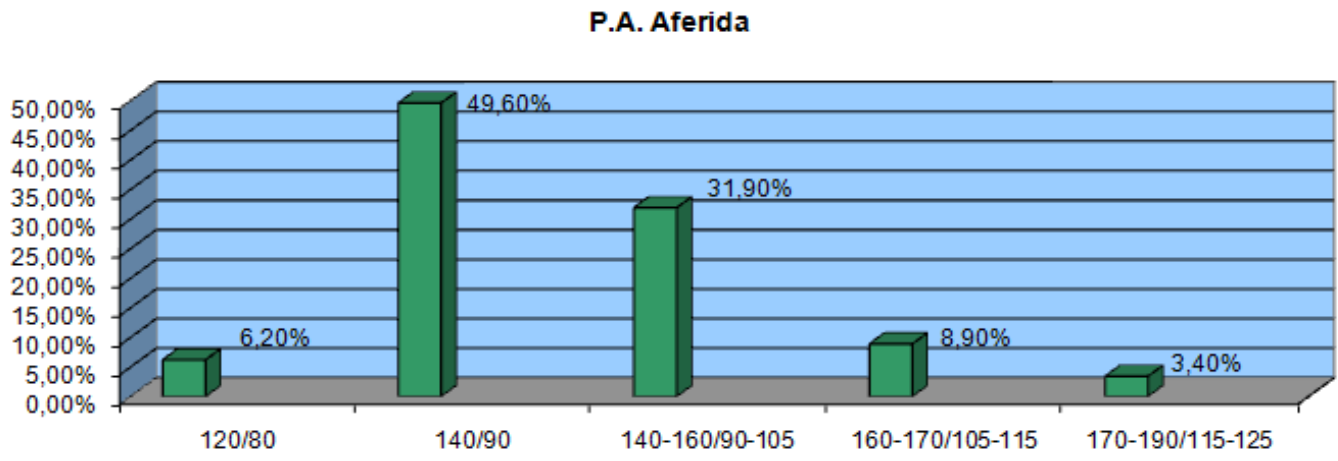

Figura 1 - Percentagem de valores de referência para pressão arterial (PA): Normal (120/80); hipertensão arterial sistêmica (HAS) Controlada (140/90); HAS Leve (140-160/90-105); HAS Moderada (160-170/105-115); HAS Grave

$(170-190 / 115-125)$

A escolaridade foi classificada em níveis, sendo de $16(14,2 \%)$ até $2^{\circ}$ grau, $82(72,6 \%)$ até $1^{\circ}$ grau e $15(13,2 \%)$ analfabetos, incluindo os funcionais (Figura 2).

\section{Escolaridade}

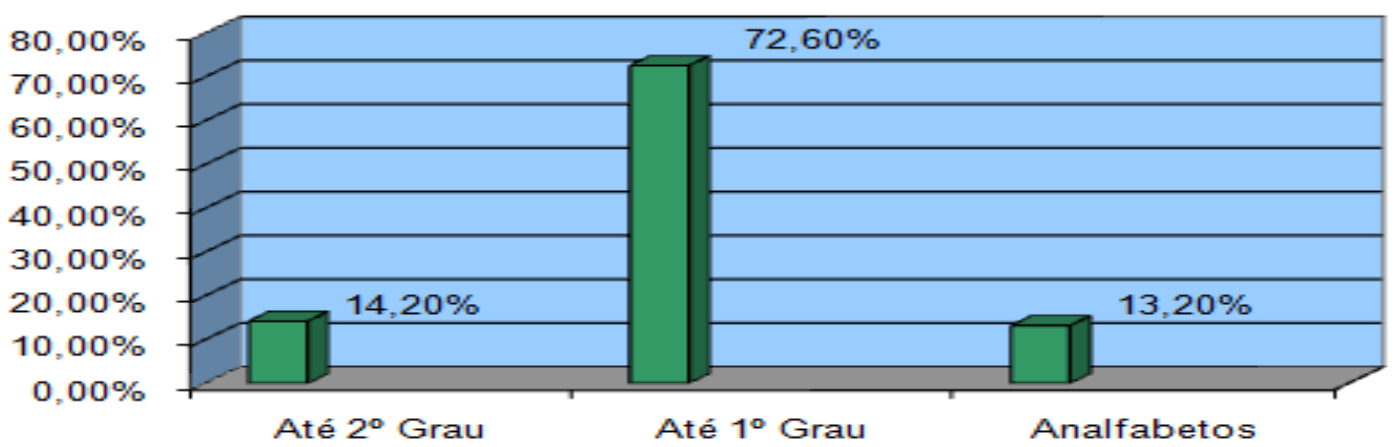

Figura 2 - Percentagem do nível de escolaridade

A média de renda foi de 79 (69,9\%) para até 1 salário mínimo (SM), 17 (15,1\%) para até 2 SM e $8(7,1 \%)$ para 2 SM ou mais. Estes, na sua maioria são aposentados, pensionistas ou trabalhadores informais. Apenas $9(7,9 \%)$ relataram não possuir nenhuma fonte de renda (Figura 3). 


\section{Renda Mensal}

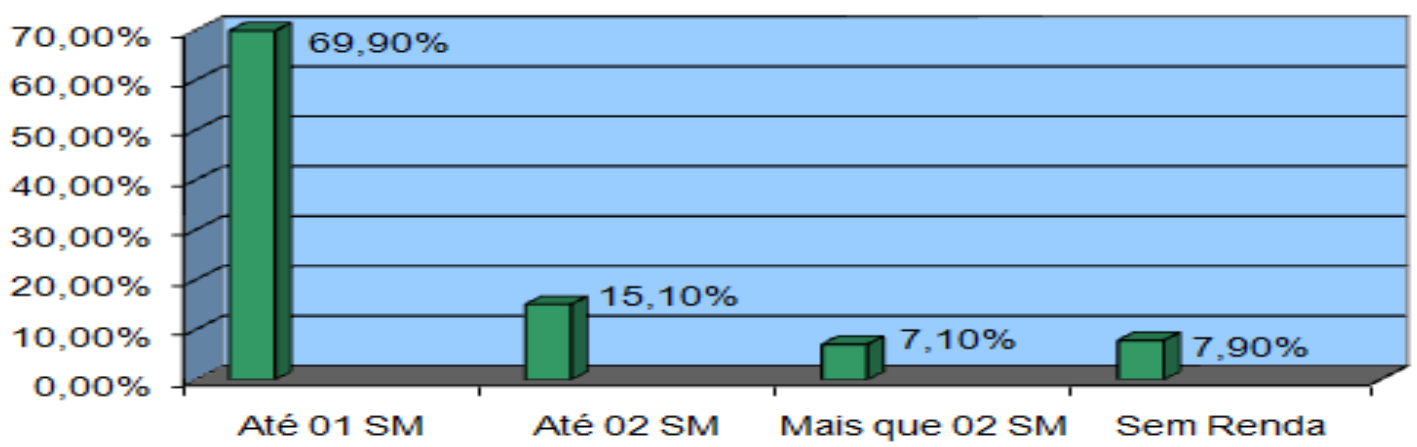

Figura 3 - Percentagem da renda mensal média, tendo como referência o salário mínimo (SM)

No total, foram examinados 870 dentes com média de 7,69 dentes por idoso. A maioria dos entrevistados se mostrou satisfeita com a capacidade mastigatória $66(58,4 \%)$ e com a aparência dos dentes/prótese 59 (52,2\%). Houve referência de diminuição no fluxo salivar em $45(39,8 \%)$ dos entrevistados e $97(85,8 \%)$ fazem uso de pelo menos um fármaco de uso diário.

Dos 113 examinados, $82(72,5 \%)$ eram desdentados parciais, 29 (25,6\%) eram desdentados totais: sendo destes $4(13,8 \%)$ não usuários de prótese e $25(86,2 \%)$ usuários de prótese. No grupo ainda foram encontrados 2 (1,9\%), com apenas restos radiculares.

Dos entrevistados 26 (23\%) relataram não usar nenhum tipo de prótese e em 87 (77\%) foi verificado o uso de próteses (Figura 4). A distribuição destas foi de 85 (60,3\%) de prótese total (PT) e $56(39,7 \%)$ de prótese parcial removível (PPR), com 61 (70,8\%) para PT superior e $24(29,2 \%)$ para PT inferior. Nas PPRs foram encontrados 24 (36,36\%) para arcada superior $32(63,64 \%)$ para inferior. A média de tempo de uso das próteses foi superior a 10 anos.

Uso de Prótese

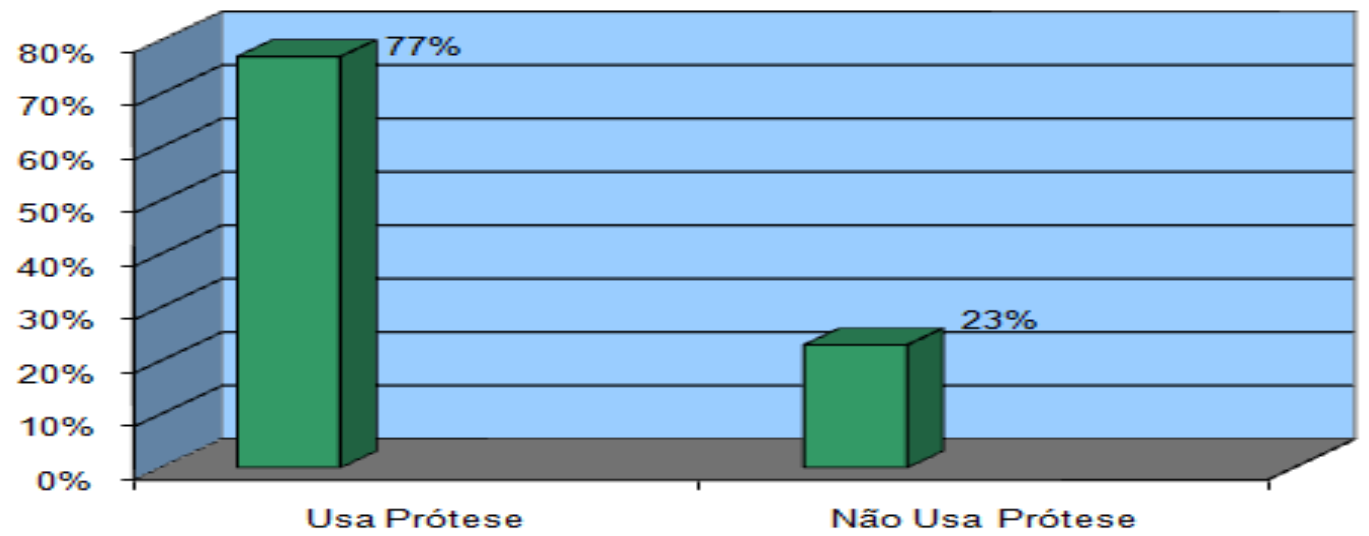

Figura 4 - Percentagem do uso de prótese 
De modo geral a higiene bucal é realizada 3 vezes ao dia por $52(46,1 \%)$ idosos, 2 vezes ao dia por $47(41,6 \%)$ e 1 vez ao dia por 14 (12,3\%). (Figura 5). A maioria não faz uso de fio dental $92(81,4 \%)$ ou de enxaguatórios bucais $95(84,07 \%)$.

Higiene Bucal

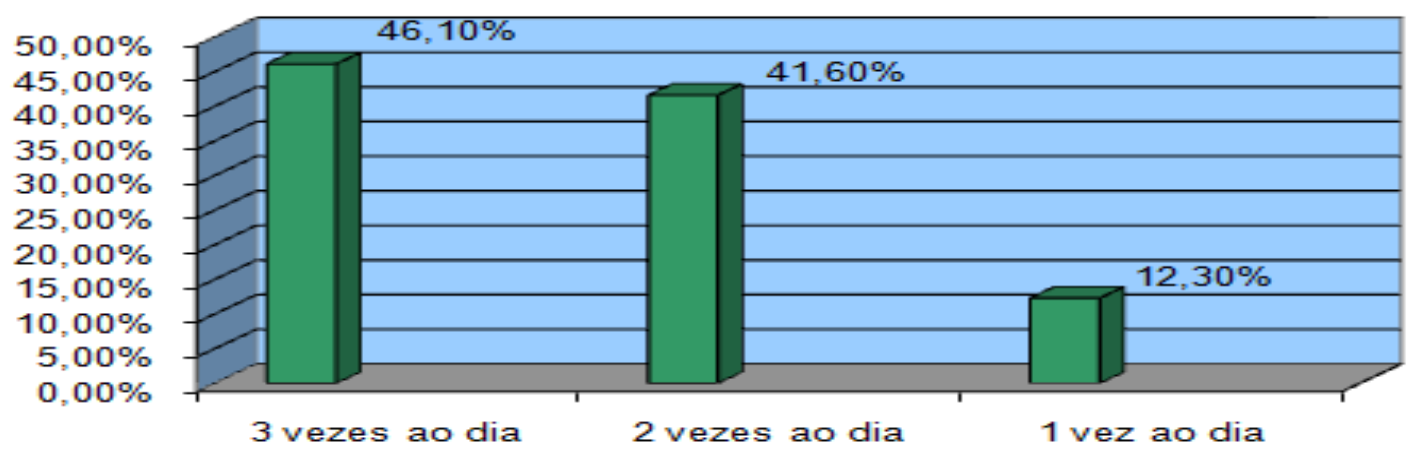

Figura 5 - Percentagem da freqüência de escovação dente/ prótese dia

No exame periodontal realizado nos 82 pacientes dentados, verificou-se em pelo menos um sitio que $46(56,1 \%)$ apresentavam $\mathrm{PS} \geq 4,24(29,3 \%)$ PS $\geq 5,10(12,2 \%)$ PS $\geq 6$ e 7 $(8,6 \%) \mathrm{PS} \geq 7$. Em relação ao NIC, encontraram-se 66 (80,5\%) com NIC $\geq 4$ e 31 (37,8\%) com NIC>6. (Figura 6).

\section{Exame Periodontal}

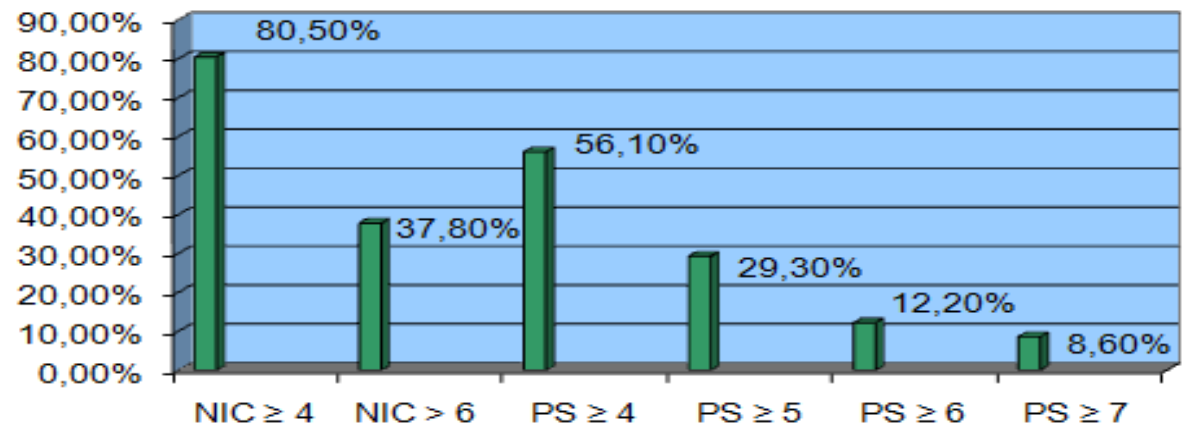

Figura 6 - Percentagem de indivíduos de acordo com valores máximos encontrados do nível de inserção clínica (NIC) e da profundidade de sondagem (PS)

\section{DISCUSSÃO}


Na maioria dos estudos, os grupos são predominantemente compostos por mulheres, isto se deve tanto à maior expectativa de vida do sexo feminino, quanto a uma maior preocupação nos cuidados com a saúde por parte dessas. Nos achados de Tanaka e Esteves, ${ }^{(1)}$ 44\% da mostra possuía renda entre 1 a 3 SM, no grupo estudado a média foi de $69,91 \%$ para até $1 \mathrm{SM}$, incluindo neste grupo os trabalhadores informais, pensionistas e aposentados. Mesmo retratando a realidade de renda da região nordeste, estes valores não mudaram de forma significativa a condição de saúde do grupo avaliado, mostrando desta forma a importância da motivação e instrução do odontólogo ao paciente.

Mesmo utilizando uma classificação diferente de Rocha et al., ${ }^{(4)}$ onde foram achados $50 \%$ de sujeitos considerados hipertensos, os resultados deste estudo mostram uma prevalência de 49,55\% para hipertensão controlada, indicando que não só o uso de medicamentos, como hábitos de vida saudáveis, dieta equilibrada e pratica de exercício físico de forma regular, contribuem para manutenção da PA em níveis satisfatórios.

Ao confrontar os dados achados no grupo institucionalizado avaliado por Innocentini e Pucca Júnior, ${ }^{(9)}$ foi achado $78 \%$ em média de necessidade do uso de prótese, isto evidencia que não há uma grande diferença, na necessidade de tratamento odontológico requerido por grupos de idosos, atendidos de forma não institucionalizada. Desta forma o estudo de Tibério et al. ${ }^{(5)}$ e Werner et al. ${ }^{(10)}$ mostram a necessidade de se realizar na avaliação específica de alguns idosos, instrumentos avaliativos como testes de estado depressivo e mini-mental-state, com o objetivo de rastrear possíveis problemas cognitivos que exigiriam mudanças no planejamento.

Nos exames periodontais observou-se uma alta prevalência de indivíduos com perda de inserção $(80,5 \%)$, sendo a forma mais severa menos prevalente $(37,8 \%)$, contudo ainda elevada. Estes achados estão de acordo com os conceitos de epidemiologia da doença periodontal, uma vez que se trata de uma população idosa, de baixo nível sócio-econômico e com hábitos de higiene bucal deficiente. A percentagem de indivíduos com bolsas periodontais foi de $56,1 \%$, sendo a percentagem de indivíduos com bolsa $\geq 5 \mathrm{~mm}$ de $29,3 \%$, com bolsa $\geq 6 \mathrm{~mm}$ de $12,2 \%$, e com bolsa $\geq 7 \mathrm{~mm}$ de $8,6 \%$. Estes valores são mais baixos que os encontrados no levantamento epidemiológico publicado por Susin et al. ${ }^{(8)}$ Provavelmente esta diferença seja justificada pelas diferenças no perfil da população que, diferentemente da do presente estudo foi predominantemente composta por homens, com alta prevalência de 
Chagas et al. Condição periodontal e hábitos de higiene bucal de idosos não institucionalizados, atendidos em grupos de convivência da cidade de Salvador.

fumantes. No estudo de Susin et al. estes dois fatores foram associados à maior ocorrência de bolsas periodontais $\geq 5 \mathrm{~mm}$ de forma generalizada.

\section{CONCLUSÃO}

No presente trabalho verificou-se que a população estudada é predominantemente feminina, de baixo nível econômico-educacional, com alto índice de edentulismo e alta prevalência de doença periodontal, sendo seus hábitos de higiene bucal assim como de cuidados odontológicos deficientes. Portanto, faz-se necessário o desenvolvimento de ações que acompanhem e permitam a esta população uma melhor qualidade de cuidados odontológicos.

\section{REFERÊNCIAS}

1. Tanaka MY, Esteves IM. Características e percepção de saúde bucal de grupos de terceira idade do município de Birigui (SP). Rev ABO Nac. 2006; 14(3): 163-171.

2. Serqueira E, Brunetti L. Eles merecem esforços redobrados. Rev. ABO Nac.2002; 10(3): 142-3.

3. Instituto Brasileiro de Geografia e Estatística. [citado 29 maio 2009]. Disponível em http://www.ibge.gov.br/home.

4. Rocha R de AC, Fernandes A, Lucas RS de CC. Doenças periodontais, dieta e distúrbios cardiovasculares em idosos não institucionalizados em Campina Grande (PB). Pesq Bras Odontoped Clin Integ. 2005; 5(2): 133-140.

5. Tibério D, Ferrari FL, Santos MTBR dos. Instrumentos avaliativos para o atendimento odontológico aos pacientes geriátricos. Rev Assoc Paul Cir Dent. 2006; 60(5): 362-5.

6. Lindhe J, Karring T, Lang NP. Tratado de periodontia clínica e implantologia oral. $4^{\mathrm{a}}$ ed. Rio de Janeiro: Guanabara Koogan; 2005.

7. Sonis ST, Fazio RC, Fang L. Princípios e prática da medicina oral. $2^{a}$ ed. Rio de Janeiro: Guanabara Koogan; 1996.

8. Susin C, Valle P, Oppermann RV, Haugejorden O, Albandar JM. Occurence and risk indicatros of increased probing depth in an adult Brazilian population. J Clin Periodontol. 2005; 32:123-9. 
9. Innocentini M de M, Pucca Júnior GA. Comparação da saúde bucal entre idosos institucionalizados nos municípios de Maringá (PR) e São José do Rio Preto (SP). Rev ABO Nac. 2006; 14(1): 37-9.

10. Werner CW de A, Leal RB, Marfinati SMPA. Ficha de exame clínico odontológico para pacientes em idade avançada. J Bras Odontogeriatria 2006; 2(5): 54-66. 